"Mircea cel Batran" Naval Academy Scientific Bulletin, Volume XIX - 2016 - Issue 1

Published by "Mircea cel Batran" Naval Academy Press, Constanta, Romania // The journal is indexed in:

PROQUEST / DOAJ / DRJI / JOURNAL INDEX / I2OR / SCIENCE LIBRARY INDEX / Google Scholar / Crossref /

Academic Keys / ROAD Open Access / OAJI / Academic Resources / Scientific Indexing Services / SCIPIO

\title{
NEW PERSPECTIVES IN THE METRIC THEORY OF CONTINUED FRACTION EXPANSION RELATED TO FIBONACCI TYPE SEQUENCES
}

\author{
Dan LASCU ${ }^{1}$ \\ Gabriela lleana SEBE ${ }^{2}$ \\ ${ }^{1}$ Lecturer Ph.D., Department of Navigation and Naval Transport, Naval Academy "Mircea cel Batran", \\ Romania, lascudan@gmail.com \\ ${ }^{2}$ Assoc.Prof. Ph.D., Faculty of Applied Sciences, Politehnica University of Bucharest, Splaiul Independentei \\ 313, 060042, Bucharest, Romania and Institute of Mathematical Statistics and Applied Mathematics, Calea \\ 13 Sept. 13, 050711 Bucharest, Romania, igsebe@yahoo.com
}

Abstract: A survey of the metric theory of the continued fraction expansions related to random Fibonacci Type sequences discussed by Sebe and Lascu is given. The limit properties of these expansions have been studied. A Wirsing-type approach to the Perron-Frobenius operator of the generalized Gauss map under its invariant measure allows us to get close to the optimal convergence rate. Actually, we obtain upper and lower bounds of the convergence rate which provide a near-optimal solution to the Gauss-Kuzmin-Lévy problem for these expansions.

Keywords: continued fractions, random Fibonacci-type sequences, Perron-Frobenius operator, random system with complete connections, Gauss-Kuzmin-Lévy problem

\section{INTRODUCTION}

In this paper we consider a non-regular continued fraction expansions introduced by Chan [1].

In fact, Chan considered some continued fraction expansions related to random Fibonacci-type sequences which were studied in detail by Sebe and Lascu in $[6,4,5]$.

PREREQUISITES. Fix an integer $m \geq 2$. Define on $I:=[0,1]$ the transformation $\tau_{m}$ by $\tau_{m}: I \rightarrow I$,

$$
\tau_{m}(x):= \begin{cases}\frac{1}{m-1}\left(\frac{1}{m^{i} x}-1\right), & x \in I_{i}, \\ 0, & x=0\end{cases}
$$

where $\quad I_{i}:=\left\{x \in I: m^{-(i+1)}<x \leq m^{-i}\right\} \quad$ for

$i \in \square:=\{0,1,2, \ldots\}$. For any $x \in(0,1)$ put

$a_{n}=a_{n}(x)=a_{1}\left(\tau_{m}^{n-1}(x)\right), \quad n \in \square_{+}:=\{1,2, \ldots\}$, with

$\tau_{m}^{0}(x)=x$ and $a_{1}=a_{1}(x)= \begin{cases}\left\lfloor\log x^{-1} / \log m\right\rfloor, & x \neq 0, \\ \infty, & x=0 .\end{cases}$

Then every $x \in[0,1)$ has an infinite expansion

$$
x=\frac{m^{-a_{1}}}{1+\frac{(m-1) m^{-a_{2}}}{1+\frac{(m-1) m^{-a_{3}}}{1+\ddots}}}:=\left[a_{1}, a_{2}, \ldots\right]_{m},
$$

where $a_{n}$ 's are non-negative integers. The numbers $p_{n}(x) / q_{n}(x)=\left[a_{1}, a_{2}, \ldots\right]_{m}$ are the $n$-th order convergent of $x \in[0,1)$. Then $p_{n}(x) / q_{n}(x) \rightarrow x, n \rightarrow \infty$. Here $p_{n}$ 's and $q_{n}$ 's satisfy for $n \in \square_{+}$the following:

$$
\begin{aligned}
& p_{n}(x)=m^{a_{n}} p_{n-1}(x)+(m-1) m^{a_{n-1}} p_{n-2}(x), n \geq 2, \\
& q_{n}(x)=m^{a_{n}} q_{n-1}(x)+(m-1) m^{a_{n-1}} q_{n-2}(x), n \geq 1,
\end{aligned}
$$

with $p_{0}(x)=0, q_{0}(x)=1, p_{1}(x)=1, q_{-1}(x)=0$ and $a_{0}=0$. In [1] it was shown that the invariant probability measure of the transformation $\tau_{m}$ is

$$
\gamma_{m}(A):=k_{m} \int_{A} \frac{d x}{((m-1) x+1)((m-1) x+m)},
$$

$A \in B_{1}$, where $B_{1}$ is the $\sigma$-algebra of the Borel subsets of $I$. Hence, $\gamma_{m}(A)=\gamma_{m}\left(\tau_{m}^{-1}(A)\right), A \in B_{1}$, the sequence $\left(a_{n}\right)_{n \in \square_{+}}$is strictly stationary on $\left(I, B_{1}, \gamma_{m}\right)$.

METRIC PROPERTIES. Roughly speaking, the metrical theory of continued fraction expansions is about the sequence $\left(a_{n}\right)_{n \in \square_{+}}$and related sequences. For any $n \in \square_{+}$and $i^{(n)}=\left(i_{1}, \ldots, i_{n}\right) \in \square^{n} \quad$ we will say that $I_{m}\left(i^{(n)}\right)=\left\{\omega \in \Omega: a_{k}(\omega)=i_{k}, 1 \leq k \leq n\right\} \quad$ is the fundamental interval of rank $n$ and make the convention that $I_{m}\left(i^{(0)}\right)=\Omega$, where $\Omega$ is the set of irrationals in $l$. We will write $I_{m}\left(a_{1}, \ldots, a_{n}\right)=I_{m}\left(a^{(n)}\right), \quad n \in \square_{+}$. If $n \geq 2$ and $i_{n} \in \square$, then we have $I_{m}\left(a_{1}, \ldots, a_{n}\right)=I_{m}\left(i^{(n)}\right)$. For any $n \in \square_{+}$we have 
"Mircea cel Batran" Naval Academy Scientific Bulletin, Volume XIX - 2016 - Issue 1

Published by "Mircea cel Batran" Naval Academy Press, Constanta, Romania // The journal is indexed in:

PROQUEST / DOAJ / DRJI / JOURNAL INDEX / I2OR / SCIENCE LIBRARY INDEX / Google Scholar / Crossref /

Academic Keys / ROAD Open Access / OAJI / Academic Resources / Scientific Indexing Services / SCIPIO

$$
\lambda\left(\tau_{m}^{n}<x \mid a_{1}, \ldots, a_{n}\right)=\frac{\left(s_{n}+m\right) x}{s_{n}+(m-1) x+1}, x \in I
$$

where $s_{n}=m^{-a_{n}} \frac{q_{n}}{q_{n-1}}-1, s_{1}=0$. Equation (4) is the Brodén-Borel-Lévy formula for this type of expansions. It allows us to determine the probability distribution of $\left(a_{n}\right)_{n \in \square_{+}}$under the Lebesgue measure $\lambda$. Clearly,

$\lambda\left(a_{1}=i\right)=(m-1) m^{-(i+1)}, i \in \square \quad$ and $n \in \square_{+} \quad$ and $\lambda\left(a_{n+1}=i \mid a_{1}, \ldots, a_{n}\right)=P_{m, i}\left(s_{n}\right)$, where

$P_{m, i}(x)=\frac{(m-1) m^{-(i+1)}(x+1)(x+m)}{\left(x+(m-1) m^{-i}+1\right)\left(x+(m-1) m^{-(i+1)}+1\right)}$.

We have already noticed that the sequence $\left(a_{n}\right)_{n \in \square}$ is strictly stationary on $\left(I, B_{1}, \gamma_{m}\right)$. As such, a doubly infinite version of it, say $\left(\bar{a}_{l}\right)_{l \in \square}$ should exist on a richer probability space. Indeed, such a version can be effectively constructed on $\left(I^{2}, B_{1}^{2}, \bar{\gamma}_{m}\right)$ where $\bar{\gamma}_{m}$, the extended measure, is defined by

$$
\bar{\gamma}_{m}(B)=k_{m} \iint_{B} \frac{d x d y}{((m-1)(x+y)+1)^{2}}, B \in B_{1}^{2} .
$$

For $n \in \square$, and $(\omega, \theta) \in \Omega^{2}$, put $\bar{a}_{n}(\omega, \theta)=a_{n}(\omega)$, $\bar{a}_{0}(\omega, \theta)=a_{1}(\theta), \bar{a}_{-n}(\omega, \theta)=a_{n+1}(\theta)$. Then for any $I \in \square, k \in \square$ and $n \in \square$, the probability distribution of the random vector $\left(\bar{a}_{l}, \ldots, \bar{a}_{l+k}\right)$ under $\bar{\gamma}_{m}$ is identical with that of the random vector $\left(a_{n}, \ldots, a_{n+k}\right)$ under $\gamma_{m}$. In other words, the doubly infinite sequence $\left(\bar{a}_{l}\right)_{l \in \square}$ is strictly stationary under $\bar{\gamma}_{m}$. The definition of $\left(\bar{a}_{l}\right)_{l \in \square}$ is associated with the natural extension $\bar{\tau}_{m}$ of $\tau_{m}$ which is a transformation of $[0,1) \times I$ defined by $\bar{\tau}_{m}(x, y)=\left(\tau_{m}(x), \frac{m^{-a_{1}(x)}}{(m-1) y+1}\right),(x, y) \in[0,1) \times I$.

This is a one-to-one transformation of $\Omega^{2}$ with the inverse

$$
\bar{\tau}_{m}^{-1}(\omega, \theta)=\left(\frac{m^{-a_{1}(\theta)}}{(m-1) \omega+1}, \tau_{m}(\theta)\right),(\omega, \theta) \in \Omega^{2} .
$$

The extended measure $\bar{\gamma}_{m}$ is $\bar{\tau}_{m}$-invariant, that is, $\bar{\gamma}_{m}=\bar{\gamma}_{m} \bar{\tau}_{m}^{-1}$ and $\overline{\boldsymbol{a}}_{l+1}(\omega, \theta)=\overline{\boldsymbol{a}}_{1}\left(\bar{\tau}_{m}(\omega, \theta)\right)$ with $\bar{a}_{1}(\omega, \theta)=a_{1}(\omega),(\omega, \theta) \in \Omega^{2}$. The dependence structure of $\left(\bar{a}_{l}\right)_{l \in \square}$ follows from the fact that $\bar{\gamma}_{m}\left([0, x] \times I \mid \bar{a}_{0}, \bar{a}_{-1, \ldots}\right)=\frac{((m-1) a+m) x}{(m-1)(x+a)+1} \quad \bar{\gamma}_{m}-$ a.s., for any $x \in I$, where $a=\left[\bar{a}_{0}, \bar{a}_{-1}, \ldots\right]_{m}$. Hence $\bar{\gamma}_{m}\left(\bar{a}_{1}=i \mid \bar{a}_{0}, \bar{a}_{-1}, \ldots\right)=P_{m, i}((m-1) a) \quad \bar{\gamma}_{m}$-a.s. for any $i \in \square$. We thus see that $\left(\bar{a}_{l}\right)_{l \in \square}$ is an infinite order chain in the theory of dependence with complete connections (see [2], section 5.5).

\section{GAUSS-KUZMIN-TYPE THEOREM}

It is only recently $[6,4,5]$ that the limits and ergodic properties of these expansions have been studied. It should be stressed that the ergodic theorem does not yield rates of convergence for mixing properties; for this a Gauss-Kuzmin theorem is needed.

Limits properties. Let us consider the random system with complete connections RSCC ([2])

$$
\left\{\left(I, B_{l}\right),\left(\square+, P_{l}\left(\square_{+}\right)\right), u, P\right\},
$$

where

$$
u: I \times \square \rightarrow I, u(x, i)=u_{m, i}(x)=\frac{m^{-i}}{(m-1) x+1} \quad x \in I
$$

and $P$ is the transition probability function from $\left(I, B_{l}\right)$ to $\left(\square_{+}, P_{l}\left(\square_{+}\right)\right)$given in (5). Here $P_{l}\left(\square_{+}\right)$ denotes the power set of $\square_{+}$. For any $a \geq 0$ put $s_{0, a}=a \quad$ and $\quad s_{n, a}=\frac{(m-1) m^{-a_{n}}}{1+s_{n a 1}^{a}}, n \in \square_{+}, \quad$ and consider the family of (conditional) probability measures $\left(\gamma_{m, a}\right)_{a}$ on $B_{1}$ defined by their distribution

$\gamma_{m, a}([0, x])=\frac{((m-1) a+m) x}{(m-1)(x+a)+1}, \quad x \in I, \quad a \geq 0$.

Then $\left(s_{n, a}\right)_{n \in \square_{+}}$is a $I \cup\{a\}$-valued Markov chain on $\left(I, B_{1}, \gamma_{m, a}\right)$ which starts from $s_{0, a}=a \geq 0$ and has the following transition mechanism: from state $s \in I \cup\{a\}$ the possible transitions are to any state $m^{-i} /((m-1) s+1)$ with the corresponding transition probability $P_{m, i}((m-1) s), \quad i \in \square$. Thus the transition operator (Perron-Frobenius operator) $U_{m}$ of all Markov chains $\left(s_{n, a}\right)_{n \in \square}$ for any bounded complex-valued measurable function $f$ on $I$, is given by 
"Mircea cel Batran" Naval Academy Scientific Bulletin, Volume XIX - 2016 - Issue 1

Published by "Mircea cel Batran" Naval Academy Press, Constanta, Romania // The journal is indexed in:

PROQUEST / DOAJ / DRJI / JOURNAL INDEX / I2OR / SCIENCE LIBRARY INDEX / Google Scholar / Crossref /

Academic Keys / ROAD Open Access / OAJI / Academic Resources / Scientific Indexing Services / SCIPIO

$$
U_{m} f(x)=\sum_{i \in \square_{+}} P_{m, i}((m-1) x) f\left(u_{m, i}(x)\right)
$$

$f \in L_{\gamma_{m}}^{1}$, where $L_{\gamma_{m}}^{1}:=\left\{f: I \rightarrow \square\left|\int_{0}^{1}\right| f \mid d \gamma_{m}<\infty\right\}$.

It was investigated in $[6,4,5]$ the PerronFrobenius operator of the continued fraction transformation $\tau_{m}$ under different probability measures on $B_{1}$. The asymptotic behavior of this operator is given by

$$
\mu\left(\tau_{m}^{-n}(A)\right)=\int_{A} \frac{U_{m}^{n} f(x)}{((m-1) x+1)((m-1) x+m)} d x
$$

for any $n \in \square$ and $A \in B_{1}$, where $f(x)=((m-1) x+1)((m-1) x+m) h(x), x \in I$. In the sequel the domain of $U$ will be successively restricted to various Banach spaces. Recall that the variation $\operatorname{var}_{A} f$ of $f$ on a subset $A$ of $l$ is defined as $\sup \sum_{i=1}^{k-1}\left|f\left(t_{i}\right)-f\left(t_{i-1}\right)\right|$ the supremum being taken over all $t_{1}<\ldots<t_{k} \in A$ and $k \geq 2$. If $\operatorname{var} f=\operatorname{var}_{l} f<\infty$ then $f$ is called a function of bounded variation. A variation $v(f)$ for $L^{\infty}\left(I, B_{1}, \lambda\right)$, the collection of all classes of $\lambda$-essentially bounded measurable complex-valued $\lambda$ indistinguishable function on $l$ is defined as $v(f)=\inf \operatorname{var} f$, the infimum being taken over all versions of . The set $B E V(I)$ is a Banach space under the norm $\|f\|_{v}:=v(f)+\|f\|_{1}$, where \|\|$_{1}$ is the usual $L_{\lambda}^{1}$ norm $\|f\|_{1}=\int_{I}|f| d \lambda$. For proofs and more details see $[4,5]$.

Whatever $a \geq 0$ the Markov chain $\left(s_{n, a}\right)_{n \in \square}$ associated with the RSCC (7) has the transition operator $U$, with the transition probability function $Q_{m}(x, A)=\sum_{i \in W_{m}(x, A)} P_{m, i}(x), \quad x \in I, \quad A \in B_{1}$, where $W_{m}(x, A)=\left\{i \in \square \mid u_{m, i}(x) \in A\right\}$. Then $Q^{n}(\cdot, \cdot)$ will denote the $n$-step transition probability function of the same Markov chain. It was proved in [4] that the RSCC (7) is uniformly ergodic and its transition operator is regular with respect to the Banach space of Lipschitz functions. Now for a probability measure $\mu$ on $B_{1}$ we may determine the limit of the sequence $\left(\mu\left(\tau_{m}^{n}<x\right)\right)_{n \in \square_{+}}$as $n \rightarrow \infty$ and obtain the rate of this convergence,

$$
\begin{aligned}
& \text { i.e., } \lim _{n \rightarrow \infty} \mu\left(\tau_{m}^{n}<x\right)=\frac{k_{m}}{(m-1)^{2}} \log \frac{m((m-1) x+1)}{(m-1) x+m}, \\
& x \in I \text {, where } k_{m}=\frac{(m-1)^{2}}{\log \left(m^{2} /(2 m-1)\right)} .
\end{aligned}
$$

A WIRSING TYPE APPROACH. Using a Wirsingtype approach [7], in [6] it was obtained a better estimate of the convergence rate involved. The strategy was to restrict the domain of the PerronFrobenius operator of $\tau_{m}$ under its invariant measure $\gamma_{m}$ to the Banach space of functions which have a continuous derivative on $I$. Define a linear operator $\quad V_{m}: C(I) \rightarrow C(I)$ by $V_{m} g=-\left(U_{m} f\right)^{\prime}, \quad g \in C(I)$, where $f^{\prime}=g$. Since $U_{m}$ is a Markov operator, $V_{m}$ is well defined. It is easy to check that $\left(U_{m}^{n} f\right)^{\prime}=(-1)^{n} V_{m}^{n} f^{\prime}, \quad n \in \square_{+}$, $f \in C^{1}(I)$. In [6] Sebe proved that there are positive constants $v_{m}<w_{m}<1$ and a real-valued function $\varphi_{m} \in C(I)$ defined by

$\varphi_{m}(x)=(m-1) e_{m}^{2}$.

$\frac{\left(m^{2}-1\right)\left((m-1)(a+1)-e_{m}\right) x^{2}+2 m\left((m-1)(a+1)+(m-2) e_{m}\right) x+m(m+1) e_{m}}{\left(\left((m-1)(a+1)-e_{m} x\right)+e_{m}\right)^{2}\left(\left((m-1)\left(e_{m}+a+1\right)-m e_{m} x\right)+m e_{m}\right)^{2}}$

$x \in I$, where the coefficient $e_{m}$ is chosen such that the equation

$$
\begin{aligned}
& E_{m}(a)=(m+1)(m-1)^{3}(a+1)^{4}- \\
& -m\left(m^{2}+2 m-1\right) e_{m}^{3}(a+1)-m(2 m-1) e_{m}^{4}=0, x \in I
\end{aligned}
$$

has a unique solution $a_{m} \in I$. For this unique acceptable $a_{m} \in I$, we have $v_{m} \varphi_{m} \leq V_{m} \varphi_{m} \leq w_{m} \varphi_{m}$ $m \in \square, m \geq 2$. Next, putting $\alpha_{m}=\min _{x \in l} \frac{\varphi_{m}(x)}{\left(f_{m}\right)^{\prime}(x)}$ and $\beta_{m}=\max _{x \in I} \frac{\varphi_{m}(x)}{\left(f_{m}\right)^{\prime}(x)}$ for any $f_{m} \in C^{1}(I)$ such that $\left(f_{m}\right)^{\prime}>0, \quad$ we get $\frac{\alpha_{m}}{\beta_{m}} v_{m}^{n}\left(f_{m}\right)^{\prime} \leq V_{m}^{n}\left(f_{m}\right)^{\prime} \leq \frac{\beta_{m}}{\alpha_{m}} w_{m}^{n}\left(f_{m}\right)^{\prime}, n \in \square_{+}$. In Theorem 5.3 in [6] there are obtained upper and lower bounds of the convergence rate, respectively $O\left(w_{n}\right)$ and $O\left(v_{n}\right)$, which provide a near-optimal solution to the Gauss-Kuzmin-Lévy problem. Let $\mu$ be a probability measure on $B_{1}$ such that $\mu \square \lambda$. For any $n \in \square_{+}$, put 
"Mircea cel Batran" Naval Academy Scientific Bulletin, Volume XIX - 2016 - Issue 1

Published by "Mircea cel Batran" Naval Academy Press, Constanta, Romania // The journal is indexed in:

PROQUEST / DOAJ / DRJI / JOURNAL INDEX / I2OR / SCIENCE LIBRARY INDEX / Google Scholar / Crossref /

Academic Keys / ROAD Open Access / OAJI / Academic Resources / Scientific Indexing Services / SCIPIO

$F_{m}^{n}(x)=\mu\left(\tau_{m}^{n}<x\right), \quad x \in I$, where $F_{m}^{0}$ is the identity map. Let

$f_{m}^{0}(x)=((m-1) x+1)((m-1) x+m)\left(F^{0}\right)^{\prime}(x), x \in I$

where $\left(F^{0}\right)^{\prime}=\frac{d \mu}{d \lambda}$. Let us recall this theorem.

THEOREM 1 (Near optimal solution to GaussKuzmin-Lévy) Let $f_{m}^{0} \in C(I)$ such that $\left(f_{m}^{0}\right)^{\prime}>0$ and let $\mu$ be a probability measure on $B_{1}$ such that $\mu \square \lambda$. For any $n \in \square$, and $x \in I$ we have

$$
\begin{aligned}
& \frac{m \alpha_{m} \min _{x \in 1}\left(f_{m}^{0}\right)^{\prime}(x)}{2 \beta_{m} k_{m}^{2}} v_{m}^{n} G_{m}\left(1-G_{m}(x)\right) \leq \\
& \leq\left|\mu\left(T_{m}^{n}<x\right)-G_{m}(x)\right| \leq \\
& \leq \frac{m(2 m-1) \beta_{m} \max _{x \in l}\left(f_{m}^{0}\right)^{\prime}(x)}{2 \alpha_{m} k_{m}^{2}} w_{m}^{n} G_{m}(x)\left(1-G_{m}(x)\right),
\end{aligned}
$$

where $G_{m}(x)=\frac{k_{m}}{(m-1)^{2}} \log \frac{m((m-1) x+1)}{(m-1) x+m}$.
For example, for $m=5$, the equation $E_{m}(x)=0$, with $e_{m}=\sqrt[3]{5}=1.709975$, has as unique acceptable solution $a=a_{m}=0.428487$. For this value of $a$, the function $\frac{\varphi_{m}}{V_{m} \varphi_{m}}$ attains its maximum equal to 3.349763 at $x=0$ and $x=1$ and has

a minimum $m(a)=\frac{\varphi_{m}}{V_{m} \varphi_{m}}(0.008438)=3.319392$. It follows that upper and lower bounds of the convergence rate are respectively $O\left(w_{5}^{n}\right)$ and $O\left(v_{5}^{n}\right)$ as $n \rightarrow \infty$, with $v_{5}>0.298528$ and $w_{5}<0.301259$. Obviously, the determination of the exact convergence rate remains an open question. We may derive it using the same strategy as in [3].

\section{BIBLIOGRAPHY}

[1] H.-C. Chan, The asymptotic growth rate of random Fibonacci type sequences. II, Fibonacci Quart. 44 (2006), 73-84.

[2] Iosifescu, M., Grigorescu, S., Dependence With Complete Connections and its Applications, Cambridge Tracts in Mathematics 96, 1990. Cambridge Univ.Press, Cambridge. [(2009): second printing slightly corrected].

[3] Iosifescu, M., Sebe, G.I., An Exact Convergence Rate in a Gauss-Kuzmin-Lévy Problem for Some Continued Fraction Expansion. In vol. Mathematical Analysis and Applications, 90-109, AIP Conf. Proc. 835, Amer. Inst. Physics, Melville, New York, 2006.

[4] Lascu, D., On a Gauss-Kuzmin-type problem for a family of continued fraction expansions, J. Number Theory 133(7) (2013), 2153-2181.

[5] Lascu, D., A Gauss-Kuzmin theorem for continued fractions associated with non-positive integer powers of an integer $m \geq 2$, The Scientific World Journal, vol. 2014 (2014), 8 pages.

[6] Sebe, G.I., Convergence rate for a continued fraction expansion related to Fibonacci type sequences, Tokyo J. Math., 33(2) (2010), 487-497.

[7] Wirsing, E., On the Theorem of Gauss-Kuzmin-Lévy and a Frobenius-Type Theorem for Function Spaces, Acta Arithmetica 24 (1974), 506-528. 